\title{
Adénome pléomorphe des glandes salivaires accessoires
} Pleomorphic adenoma of minor salivary gland

\section{Résumé}

\section{MOTS-CLEFS :}

- Adénome pléomorphe, glandes salivaires accessoires, récidive

\section{KEYWORDS:}

- Pleomorphic adenoma, minor salivary gland imaging, recurrence
AOS 2015;271:4-9

DOI: $10.1051 /$ aos/2015107 (C) EDP Sciences 2015
Objectif : L'adénome pléomorphe est la variété la plus fréquente des tumeurs bénignes des glandes salivaires accessoires. Le but de ce travail est une analyse épidémiologique, clinique et thérapeutique de cette tumeur.

Matériel et méthodes : On rapporte à travers une étude rétrospective descriptive 7 cas d'adénomes pléomorphes des glandes salivaires accessoires sur une période de 7 ans (2006 à 2012).

Résultats : Il s'agissait de 1 homme et 6 femmes ayant un âge moyen de 36,85 ans. Dans trois cas, le siège de ces adénomes était le palais osseux, dans deux cas au niveau du voile, dans deux cas aux lèvres. Le délai moyen de diagnostic était de 3 ans et 10 mois. La tuméfaction a été la manifestation clinique observée dans tous les cas. La TDM a été réalisée dans tous les cas, associée ò̀ l'IRM dans 2 cas. Le traitement était chirurgical dans tous les cas. L'histologie a confirmé le diagnostic d'adénome pléomorphe. Il était bénin dans tous les cas.

La récidive n'a pas été observée sur un recul de 3 ans et 6 mois.

Conclusion : Les adénomes pléomorphes des glandes salivaires accessoires ont une évolution lente. Ils doivent être évoqués devant toute formation tumorale indolore de la muqueuse buccale, développée sous une muqueuse saine. Une exérèse tumorale avec une marge de $5 \mathrm{~mm}$ de tissu sain est le traitement adéquat. Les risques de récidive et de transformation maligne imposent une surveillance prolongée.

\author{
Abstract
}

Objective: Pleomorphic adenoma (PA) is the most common benign neoplasm of the minor salivary glands. A retrospective epidemiologic, clinical and therapeutic analysis is conducted.

Materials and methods: This is a descriptive and retrospective study about 7 cases of pleomorphic adenoma conducted over a period of 7 years (2006 ò 2012).

Results: There were 1 man and 6 women having a mean age of 36,85 years. In 3 cases, the site of this adenoma was the hard palate. Three tumors were seen in velar, in lips 2 cases where observed. Clinical examination showed a swelling at the level of the interested region. Computed tomography (CT) was made in all cases, associated to the magnetic resonance imaging (MRI) in 2 cases. The treatment was based in surgical excision of the tumors. Histologically, the tumor showed features of a benign PA in all cases. We did not observe any local recurrence.

Conclusion: Pleomorphic adenoma of minor salivary gland is generally known to be a slowgrowing tumour. They must be evoked for each tumor developed under a healthy oral mucosa. The surgical management is basically total resection of the tumor with a safety margin of $5 \mathrm{~mm}$. Longer follow-up was needed due to the possibility of recurrences and malignancy transformation.

Jalal HAMAMA', Docteur en médecine et spécialiste en chirurgie maxillo-faciale
et stomatologie,
Saida EL KHAYATI ', Docteur en chirurgie dentaire
Adil ARROB ${ }^{1}$, Docteur en médecine et spécialiste en chirurgie plastique
Lahcen KHALFI', Docteur en médecine et spécialiste en chirurgie maxillo-faciale
et stomatologie
Abdeljalil ABOUCHADI ${ }^{2}$, Docteur en médecine et professeur agrégé en chirurgie
maxillo-faciale et stomatologie
Mohammed Karim ELKHATIB ${ }^{2}$, Docteur en médecine et professeur agrégé de Val
de Grace en chirurgie plastique. Chef de service
'Service de stomatologie et de chirurgie plastique. Hôpital militaire d'instruction Mohammed V,
Rabat-royaume du Maroc.
'Service de stomatologie et de chirurgie plastique - Hôpital militaire d'instruction Mohammed $\mathrm{V}$
Rabat-Royaume du Maroc. Université Mohammed V. Faculté de médecine et de pharmacie,
Rabat-Royaume Du Maroc.




\section{INTRODUCTION}

Les tumeurs des glandes salivaires accessoires (GSA) sont rares et réputées être bénignes dans la majorité des cas. Toutefois, leur chef de file qui est l'adénome pléomorphe (AP) demeure un souci majeur pour tout chirurgien maxillo-facial à cause de son risque de dégénérescence maligne. Ainsi il nous a paru judicieux d'apprécier la qualité de notre prise en charge à travers une étude rétrospective.

\section{MATÉRIEL ET MÉTHODES}

Sept patients atteints d'AP des GSA ont été pris en charge entre 2006 et 2012 au service de stomatologie et de chirurgie maxillo-faciale de l'hôpital militaire d'instruction Mohammed V de Rabat. Les dossiers médicaux ont été revus rétrospectivement. Tous ces patients ont bénéficié d'un examen clinique, d'une exploration par TDM et IRM, ils ont été opérés par la même équipe chirurgicale et les pièces opératoires ont été étudiées par le même laboratoire d'anatomie pathologique.

\section{RÉSULTATS}

Il s'agissait de 7 patients, 1 homme et 6 femmes. Lâge moyen était de 36,85 ans avec des extrêmes allant de 19 ans à 70 ans.

Trois cas portaient sur une tumeur du palais dur, leur âge moyen était de 49 ans avec une prédominance féminine. Ces personnes avaient consulté pour une tuméfaction palatine (fig. 1) d'un diamètre moyen de $4 \mathrm{~cm}$, indurée, indolore, bien limitée, recouverte d'une muqueuse normale avec un délai moyen dévolution de 7,6 ans. Le reste de l'examen clinique ORL et cervico-facial était tout à fait normal. Le scanner pratiqué a conclu à un processus tumoral du palais de densité tissulaire homogène avec des limites nettes et régulières et un rehaussement modéré par le produit de contraste associé à un amincissement de l'os en regard.

La localisation au niveau du voile a été observée dans deux cas dont un adolescent de 19 ans (fig. 2), leur âge moyen était de 25,5 ans. Cette localisation se manifestait par un nodule enchâssé dans l'hémivoile avec un délai moyen dévolution de 2,5 ans.

L'imagerie par résonance magnétique a objectivé un hyposignal $\mathrm{T} 1$ et un hypersignal $\mathrm{T} 2$ intense, après injection de gadolinium, et un rehaussement modéré du signal en périphérie. (fig. 3).

Chez deux femmes dont lâge moyen était de 30 ans, la localisation était au niveau de la lèvre inférieure,

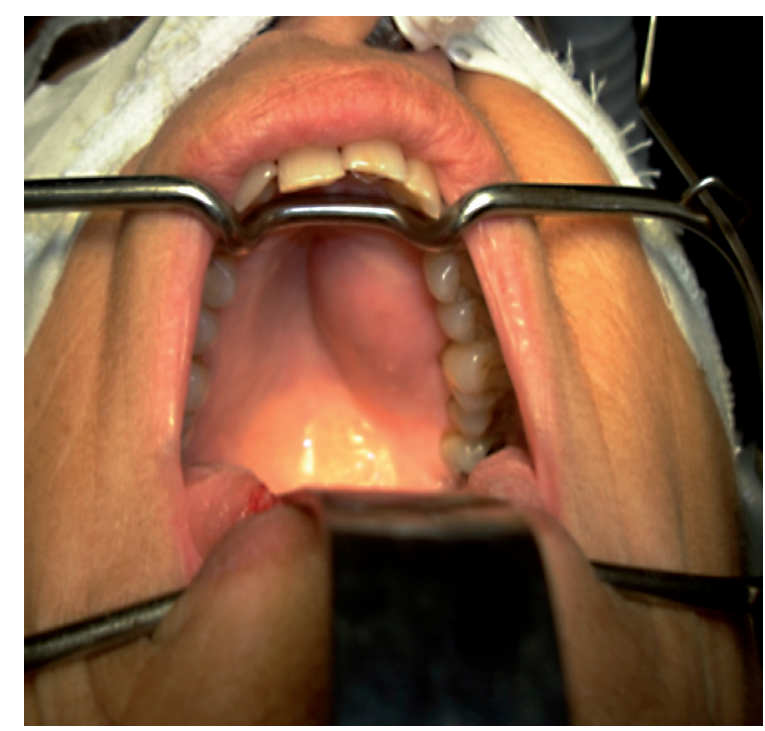

$\triangle$ Fig. 1 : adénome pléomorphe du palais dur.

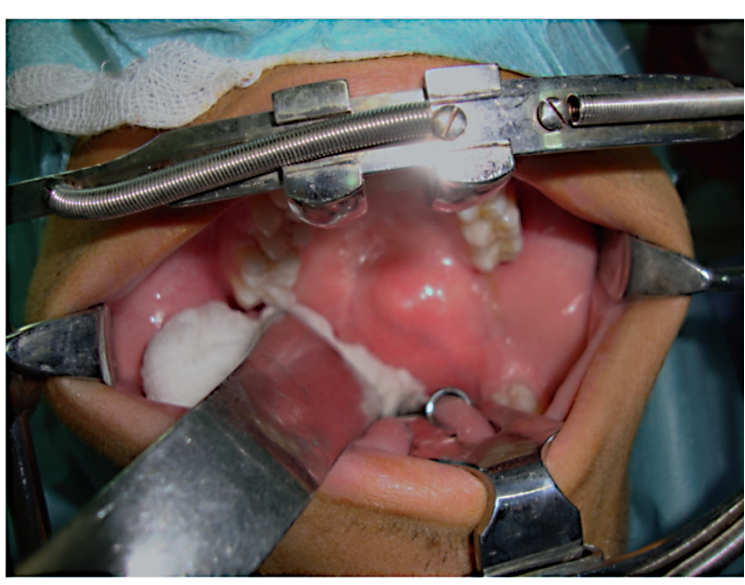

$\triangle$ Fig. 2 : adénome pléomorphe du palais mou.

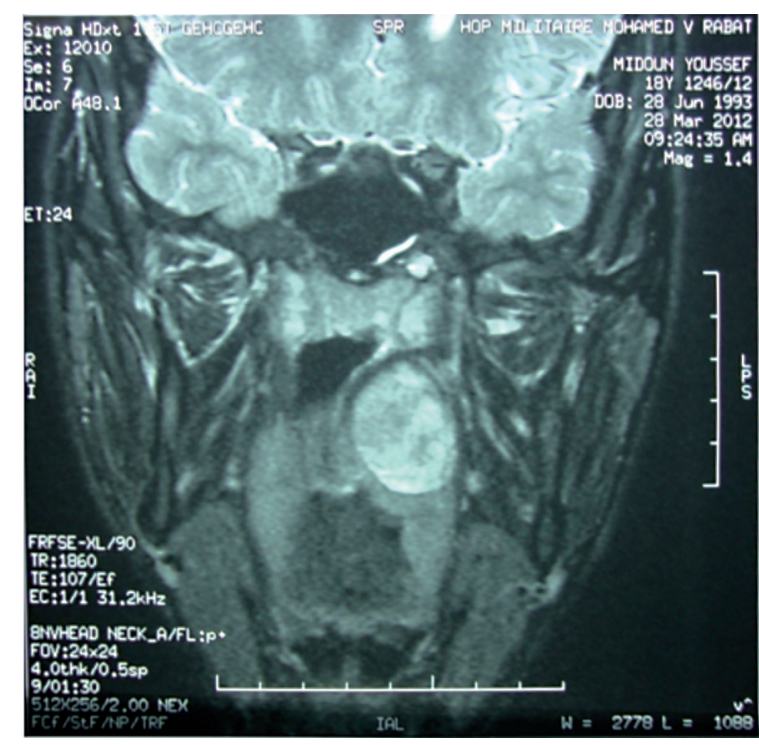

$\triangle$ Fig. 3 : IRM en coupe coronale montrant un processus ovalaire de $45 \mathrm{~mm} \times 35 \mathrm{~mm}$ en hypersignal T2 bien limité rehaussé de façon hétérogène après injection de Gadolinium,qui bombe en arrière dans l'oropharynx et se continue dans la région tonsillaire droite. 


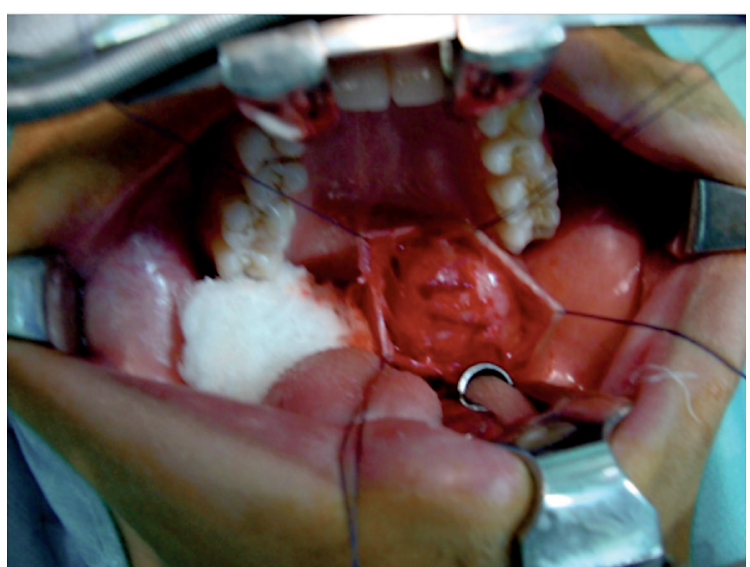

$\triangle$ Fig. 4 : aspect per opératoire d'un adénome pléomorphe.

qui s'est manifestée par un nodule, dur, bien limité et plus proche de la muqueuse que de la peau tardivement voussurée. Le délai moyen de découverte était de 4,5 mois.

Nous avons effectué l'exérèse chirurgicale de ces masses sous anesthésie générale par voie endobuccale (fig. 4). L'examen anatomopathologique a confirmé le diagnostic d'adénome pléomorphe : bénin et encapsulé dans tous les cas. Le recul moyen était de 3 ans 6 mois, aucune récidive n’a été observée.

\section{DISCUSSION}

Les tumeurs des glandes salivaires accessoires représentent $15 \%$ à $20 \%$ des tumeurs des glandes salivaires $[1,2]$.

Ladénome pléomorphe est la tumeur la plus commune $(50 \%)$ des glandes salivaires principales et accessoires [3]. Approximativement, $80 \%$ des AP se développent au niveau de la parotide, $8 \%$ au niveau de la glande submandibulaire et $7 \%$ au niveau des GSA. Il représente le type histologique le plus fréquent $(70,6$ à $100 \%$ ) des tumeurs bénignes des glandes salivaires accessoires avec un siège de prédilection au niveau du palais [4].

LAP des GSA affecte plus souvent la femme que l'homme avec des ratios variables selon les différentes études $1 / 1,1$ [5] et 1/3,2 [6]. Dans notre série, le sex-ratio était de 0,16 . Lâge préférentiel de survenue de l'AP varie entre 30 et 40 ans [7]. Il était de 36 ans pour nos malades.

Dans la tranche d'âge pédiatrique, les tumeurs des glandes salivaires sont extrêmement rares [8-11]. En effet, la localisation palatine chez des enfants de moins de 14 ans a été décrite uniquement dans cinq publications de toute la littérature anglaise [12-15] ; dans notre série, nous avons rapporté un seul cas.
La symptomatologie clinique dépend de la taille et de la localisation tumorale [16]. Au niveau de la cavité orale, on décrit souvent une tuméfaction indolore évoluant sous une muqueuse normale [17-18].

LAP du palais est souvent de siège postérolatéral, bridé entre la voûte osseuse et la fibromuqueuse épaisse et saine, la tumeur maligne déforme la région en « verre de montre ".

L'AP du voile du palais se manifeste par un nodule enchâssé dans l'hémivoile. Parfois, le nodule dévie la luette ou accapare l'épaisseur du pilier antérieur de l'amygdale.

L'AP des lèvres se manifeste par un nodule, dur, bien limité et plus proche de la muqueuse que de la peau tardivement voussurée.

Dans la plupart des cas, comme dans le nôtre, la symptomatologie clinique est pauvre car ces tumeurs bénignes sont de croissance lente et ne sont découvertes que lorsqu'elles deviennent volumineuses. Le délai de diagnostic est très variable, il est de 39 mois dans ce travail avec des extrêmes allant de 2 mois à 20 ans.

L'aspect à l'IRM dépend de la composition cellulaire et myxoïde de la tumeur. Cette tumeur est souvent lobulée, bien limitée, en hyposignal T1 et hypersignal T2, se rehaussant de façon homogène après injection de produit de contraste [19].

La cytoponction à l'aiguille permet souvent d'apporter un diagnostic rapide et fiable pour un cytopathologiste entraîné. La sensibilité varie de 73 à $93 \%$ et la spécificité de 85 à $98 \%$ selon les séries [20]. Il existe également un intérêt économique, avec un coût faible, et une réduction du nombre d'interventions chirurgicales si cet examen conforte le chirurgien dans un diagnostic de bénignité. Le cytodiagnostic préopératoire permettrait ainsi une économie de $25 \%$ par patient pris en charge pour un nodule des glandes salivaires [21]. Lexamen peropératoire, bien qu'il soit très controversé dans les pays anglo-saxons, garde sa place dans la prise en charge des tumeurs des glandes salivaires. En effet, avec une sensibilité de $74 \%$, une spécificité de $99 \%$, un taux de faux négatifs de $3,5 \%$ et un taux de faux positifs de $0,83 \%$, l'examen extemporané est une technique fiable pour différencier les tumeurs malignes des bénignes quand un diagnostic préopératoire n’a pas pu être posé. Cependant, le diagnostic de sous-type histologique est parfois plus hasardeux, compte tenu du grand polymorphisme architectural des tumeurs des glandes salivaires. L'examen extemporané garde aussi son intérêt pour l'analyse des marges opératoires [22].

Au stade clinique, le diagnostic différentiel se pose avec toutes les tumeurs bénignes de la muqueuse buccale ayant un aspect nodulaire : 
tumeurs conjonctives : fibrome; lipome; myxome ; I tumeurs dysembryoplasiques : nodule thyroïdien ectopique ; kyste du tractus thyréoglosse ; kystes dermoïdes et épidermoïdes ; kyste lymphoépithélial ; $\checkmark$ tumeurs musculaires : léiomyomes; rhabdomyome ; Itumeurs nerveuses : schwannomes ; neurinome ; névrome d'amputation; tumeur d'Abrikossoff.

Macroscopiquement, la tumeur est nodulaire, bien circonscrite voire encapsulée par une gangue conjonctive, elle est habituellement de couleur grise blanchâtre, par endroit translucide à la coupe. Sa consistance est variable, ferme ou molle et gélatiniforme. Le caractère pléomorphe fait référence à une grande richesse architecturale contrastant avec le monomorphisme des cellules épithéliales et myoépithéliales qui la composent. Celles-ci sont en effet le plus souvent régulières et « rassurantes » sur le plan cytologique. Un des éléments importants du diagnostic est l'observation d'un stroma particulier qui, de façon très caractéristique, prend un aspect myxoïde, avec parfois une différenciation cartilagineuse ou osseuse [23, 24]. Des secteurs très cellulaires sont parfois observés (on parle alors « d'adénome pléomorphe cellulaire »). Une capsule est le plus souvent observée, sauf parfois dans les adénomes pléomorphes dont le stroma est essentiellement mucoïde ou dans les localisations buccales ou nasales [25]. Des effractions capsulaires, une capsule incomplète, des pseudopodes et des nodules satellites sont fréquents [26], mais ne constituent pas pour autant des critères de malignité. Cependant, ils rendent difficile l'appréciation de la qualité de la résection tumorale et semblent faciliter la récidive locale.

Au stade deétude histologique, des problèmes diagnostiques peuvent se rencontrer dans les cas suivants :

$\checkmark$ certains AP qui ont une composante épithéliale prédominante risquent d'être confondus avec les carcinomes mucoépidermoïdes ;

$\checkmark$ d'autres, très riches en cellules myoépithéliales, ont une composante épithéliale réduite et peuvent passer inaperçus ; cette éventualité est particulièrement fréquente dans l'AP du palais ;

Ide même, certains aspects palissadiques des noyaux peuvent orienter à tord vers le diagnostic de schwannome ;

Idevant une prolifération dense des cellules indépendantes fusiformes, le diagnostic d'une tumeur maligne conjonctive peut alors se poser.

Par conséquent, l'immunohistochimie apporte une aide précieuse pour élucider ces cas difficiles.

Les marqueurs myoépithéliaux de référence sont ceux qui indiquent une différenciation musculaire, en particulier l"« actine musculaire lisse ». On peut également utiliser l'anti-vimentine ou l'anti-PS100 qui sont plus sensibles mais qui ne sont pas spécifiques des cellules myoépithéliales.

Les cytokératines CK14, CK5 et la protéine p63 reconnaissent à la fois les cellules myoépithéliales et les cellules basales. La protéine p63 est également exprimée dans la couche basale des épithéliums malpighiens et dans les carcinomes épidermoïdes.

Les anticorps " anti-Epithelial Membrane Antigene (EMA), Antigène Carcino-Embryonnaire (ACE), anti-CK7 et anti-CK19 » font ressortir les structures ductales, en particulier quand elles sont dissimulées dans un carcinome adénoïde kystique de forme massive, dans un adénome pléomorphe cellulaire, dans un carcinome épithélial myoépithélial massif. L'« anti-CD 117 (c-kit) » visualise également les cellules luminales et les ébauches de formations glandulaires. Contrairement à ce qui avait été initialement rapporté, le marquage n'est pas spécifique d'un type tumoral particulier [27].

Dans $70 \%$ des adénomes pléomorphes, on retrouve les aberrations suivantes $[28,29]$ :

$\checkmark$ le réarrangement $8 q 12$ (39\%). Le gène cible est PLAG1, un facteur de transcription qui est dérégulé lors de sa fusion avec un autre chromosome, en général après translocation, plus rarement suite à un réarrangement intrachromosomique. Pour les translocations, le gène partenaire peut être le gène de la caténine, le gène du leukemia inhibitory factor (LIFR) ou le gène du facteur de transcription et d'élongation SII.

Pour les remaniements intrachromosomiques cryptiques $8 \mathrm{q}$, les partenaires sont $\mathrm{CHCHD7}$ ou TCEA1 ; $\checkmark$ le réarrangement $12 \mathrm{q} 13-15(8 \%)$. Le gène cible est HMGA2 qui code pour une protéine de la chromatine, non histone.

Les gènes partenaires sont fragile histidine triad gene dans la translocation $\mathrm{t}(3 ; 12)(\mathrm{p} 14.2 ; \mathrm{q} 145)$ et NFIB dans l'insertion ins $(9 ; 12)$ (p23;q12q15). Les gènes de fusion aboutissent à une séparation de deux domaines de la protéine : les zones de liaison à l'ADN sont distantes des motifs de déstabilisation de l'ARNm, ce qui conduit à une hyperproduction de HMGA2 ;

$\checkmark$ les remaniements clonaux sporadiques à l'exclusion des modifications impliquant 8q21 et 12q13-15 (23\%). Les translocations de PLAG1 et HMGA2 ont été décrites uniquement dans les adénomes pléomorphes et correspondraient donc à une "signature moléculaire » de ce diagnostic. Dans les cas de diagnostic difficile, la détection de ces réarrangements par RT-PCR ou FISH pourrait être très utile.

Le traitement est essentiellement chirurgical. En effet, quel que soit le siège, l'exérèse de l'AP doit en principe être conduite à distance, une énucléation n'est pas un geste chirurgical plus convenable à ce niveau. 


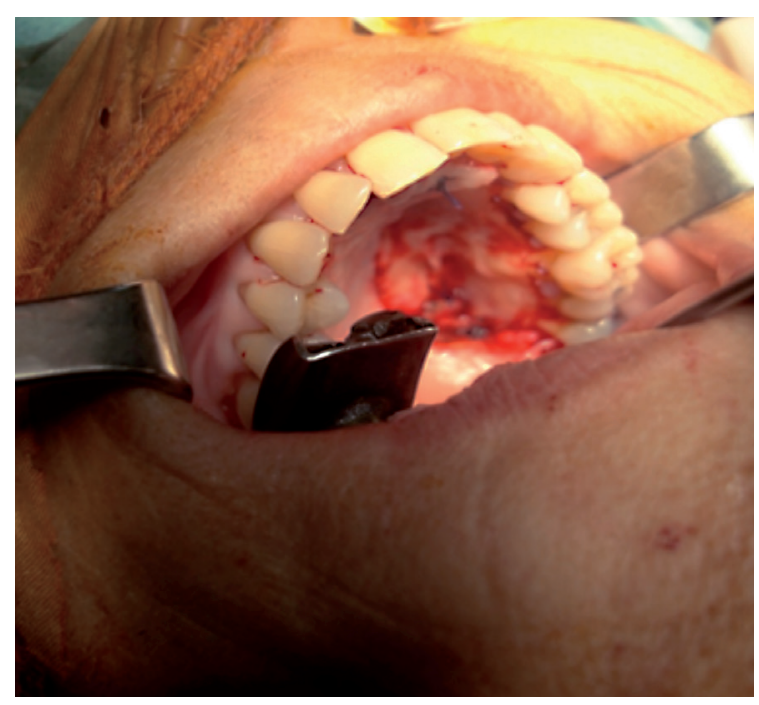

$\triangle$ Fig. 5 : aspect per opératoire montrant l'exérèse de la muqueuse palatine.

Toute incision directe avec levée de lambeau muqueux, afin de permettre une suture en fin d'intervention, est à éviter également vu le risque de laisser dans l'épaisseur de la muqueuse quelques îlots tumoraux. Par conséquent, l'exérèse de la tumeur impose le sacrifice délibéré du revêtement muqueux (fig. 5) afin déviter la récidive qui dépend du siège et de la qualité d'exérèse tumorale [30]. La littérature rapporte une fréquence de 2,4 à $10 \%$ [31].

L'incidence d'une transformation maligne ou de carcinome ex-adénome pléomorphe (CXAP) est rare. Elle survient dans moins de $7 \%$ des AP sur GSA, siégeant essentiellement au niveau du palais [32]. Le risque de transformation augmente souvent avec la fréquence des récurrences et le délai du diagnostic, varie de 1,6\% avant 5 ans à $9,4 \%$ après 15 ans [33]. L'augmentation récente de taille, l'ulcération, l'infiltration et le saignement spontané sont des signes en faveur d'une transformation maligne. L'analyse de la pièce opératoire doit être rigoureuse car la composante maligne peut être très minime [34]. La composante épithéliale dégénérée correspond le plus souvent à un adénocarcinome ou à un carcinome indifférencié.

On parle d'adénome pléomorphe métastasiant devant un adénome pléomorphe d'aspect histologique complètement bénin mais qui saaccompagne d’une dissémination locorégionale ou à distance. Cette dissémination semble être secondaire à des récidives multiples et/ou des interventions chirurgicales répétées qui permettent à la tumeur d’accéder au réseau vasculaire veineux [35]. Ces localisations secondaires, généralement osseuses, pulmonaires et ganglionnaires, surviennent jusquà 55 ans après l'adénome pléomorphe initial $[23,24]$. Le carcinome ex-(sur)adénome pléomorphe est un carcinome survenant sur un adénome pléomorphe préexistant. Tous les types histologiques de carcinomes peuvent être observés [36]. Il s’agit d'un diagnostic difficile, notamment lors d'un examen extemporané, car la composante maligne peut être très minime. Ce problème inhérent à léchantillonnage doit faire réaliser de très nombreux prélèvements pour l'examen histologique définitif, afin de ne pas méconnaittre un foyer transformé. Le diagnostic différentiel avec un adénome pléomorphe cellulaire peut être difficile quand le carcinome exadénome pléomorphe est très bien différencié. Alors que létude immunohistochimique n’a aucun intérêt dans les adénomes pléomorphes, son utilisation dans les carcinomes ex-adénomes pléomorphes peut être justifiée, en particulier pour déterminer l'index de prolifération de la composante maligne.

La progression tumorale des carcinomes ex-adénome pléomorphe semble se faire selon trois étapes successives [37]. Une dysfonction de la protéine antioncogène P53 est probablement à lorigine de ces lésions [38]. Conceptuellement, on peut définir trois groupes de tumeur au pronostic différent en fonction de leur niveau d'infiltration :

$\checkmark$ les carcinomes intracapsulaires, limités à l'adénome pléomorphe préexistant ;

$\checkmark$ les carcinomes à invasion minime, sétendant au-delà de la capsule sur une distance inférieure à $1,5 \mathrm{~mm}$ selon l'OMS ;

$\checkmark$ les carcinomes invasifs.

La survie est entre 18 et $50 \%$ à dix ans.

\section{CONCLUSION}

Notre prise en charge reste globalement satisfaisante en raison de la stratégie que nous suivons articulée sur : $\checkmark$ lévocation d'AP des GSA devant toute formation tumorale de la cavité buccale développée sous une muqueuse saine ;

$I$ la proscription de la biopsie ;

$\checkmark$ lexérèse tumorale systématique avec marge de $5 \mathrm{~mm}$ de tissu sain ;

I la surveillance qui est prolongée et rigoureuse. 


\section{Bibliographie}

[1] Varghese BT, Sebastian P, Abraham EK, Mathews A. Pleomorphic adenoma of minor salivary gland in the parapharyngeal. World J Surg Oncol 2003;1(1):2.

[2] Becelli R, Frati R, Cerulli G, Perugini M, Frati A, Lannetti G. Pleomorphic adenoma of the minor salivary glands of the palate. $J$ Exp Clin Cancer Res 2001;20(1):25-8.

[3] Clauser L, Mandrioli S, Dallera V, Sarti E, Galie M, Cavazzini L. Pleomorphic adenoma of the palate, J Craniofac Surg 2004;15(6):1026-9.

[4] Toida M, et al. Intraoral minor salivary gland tumors: a clinicopathological study of 82 cases. Int J Oral Maxillofac Surg 2005;34(5):528-32.

[5] Eveson JW, Cawson RA. Tumours of the minor (oropharyngeal) salivary glands: a demographic study of 336 cases. J Oral Pathol 1985;14(6):500-9.

[6] Isacsson G, Shear M. Intraoral salivary gland tumors: a retrospective study of 201 cases. J Oral Pathol 1983;12:57-62.

[7] Pires FR, Pringle GA, Almeida OP, Chen SY. Intra-oral minor salivary gland tumors: A clinicopathological study of 546 cases. Oral Oncol 2007;43:463-70.

[8] Callender DL, Frankenthaler RA, Luna MA, Lee SS, Goepeert H. Salivary gland neoplasm in children. Arch Otolaryngol Head Neck Surg 1992;118:472-6.

[9] LAcr EE, UvroN ME. Histopathologic review of salivary gland tumors in childhood. Arch Otolaryngol Head Neck Surg 1988;114:898-906.

[10] Luna MA, Batsakis JG, El-Naggar AK. Salivary gland tumors in children. Ann Otol Rhinol Laryngol 1991;100(10):869-71.

[11] Pogrel A. The management of salivary and tumors of the palate. J Oral Maxillofac Surg 1994;52(5):454-9.

[12] Austin JR, Crockett DM. Pleomorphic adenoma of the palate in a child. Head Neck 1992;14(1):58-61.

[13] Bvars LT, Ackeman LV, Peacock E. Tumors of salivary gland origin in children. Ann Surg 1956:146:40-51.
[14] Fonseca I, Martins AG, Soares J. Epithelial salivary gland tumors of children and adolescents in southern Portugal. $A$ clinicopathological study of twenty-four cases.

[15] Noghreyan A, Gatot A, Moar E, Fliss DM. Palatal pleomorphic adenoma in a child. J Laryngol Otol 1995: 109: 343-5.

[16] Just P-A, Miranda L, ElouaretY, Meatchi T, Hans S, Badoual C.Classification des tumeurs des glandes salivaires. Ann Otolaryngol Chir Cervicofac 2008;125:331-40.

[17] Pires FR, Pringle GA, Almeida OP, Chen SY. Intra-oral minor salivary gland tumors: A clinicopathological study of 546 cases. Oral Oncol 2007;43:463-70.

[18] Arsheed H, Biswajyoti H, Sultan A P, Rajan K. Primary pleomorphic adenoma of minor salivary gland in the parapharyngeal space. World J Surg Oncol 2009;7:85.

[19] Bullerdiek J, Wobst G, Meyer-Bolte K, Chilla R. Cytogenetic subtyping of 220 salivary gland pleomorphic adenomas. Cancer Genet Cytogenet 1993;65:27-31.

[20] Baglin AC, Wassef M. Cytoponction des glandes salivaires : le pour et le contre. Ann Pathol 2007;27:1S78-11S.

[21] Layfield LJ, Gopez E, Hirschowitz S. Cost efficiency analysis for fine-needle aspiration in the workup of parotid and submandibular gland nodules. Diagn Cytopathol 2006;34:734-8.

[22] Badoual C, et al. Evaluation of frozen section diagnosis in 721 parotid gland lesions. Histopathology 2006;49(5):538-40.

[23] Barnes L, et al. World Health Organization classification of tumours. Pathology and genetics of head and neck tumours. Lyon: IARC Press, 2005

[24] Ellis GL, Auclair PL. Atlas of tumor pathology. Third series, fascicle 17. Tumors of the salivary glands. Washington: Armed forces institute of pathology, 1996

[25] Uro-Coste E. Particularité des tumeurs des glandes salivaires accessoires. Ann Pathol 2007;27:1S76-11S.

[26] Zbären P, Stauffer E. Pleomorphic adenoma of the parotid gland: histopathologic analysis of the capsular characteristics of 218 tumors. Head Neck 2007;29:751-7.
[27] Edwards PC, Bhuiya T, Kelsch RD. C-kit expression in the salivary gland neoplasms adenoid cystic carcinoma, polymorphous lowgrade adenocarcinoma, and monomorphic adenoma. Oral Surg Oral Med Oral Pathol Oral Radiol Endod 2003;95:586-93.

[28] Kas K, Voz ML, Roijer E, et al. Promoter swapping between the genes for a novel zinc finger protein and beta-catenin in pleiomorphic adenomas with $\mathrm{t}(3 ; 8)(\mathrm{p} 21 ; \mathrm{q} 12)$ translocations. Nat Genet 1997;15(2):170-4.

[29] Michal M, et al. Cribriform adenocarcinoma of the tongue: a hitherto unrecognized type of adenocarcinoma characteristically occurring in the tongue. Histopathology 1999;35(6):495-501.

[30] Bardwill JM, Reynolds CT, Ibanez ML, Luna MA. Report of 100 tumors of minor salivary glands. Am J Surg 1966;112(4):493-7

[31] Alper C, Fatih C, Aylar P, Sabri U. Pleomorphic adenoma of the nasal columella. Pathol Res Pract 2008;204:273-6.

[32] Akan H, Yildiz L, Unal R. Carcinoma ex pleomorphic adenoma of the minor salivary gland with pulmonarymetastasis. Diagn Interv Radiol 2008;14(1):3-5.

[33] McNamara ZJ, Batstone M, Farah SC. Carcinoma ex pleomorphic adenoma in a minor salivary gland of the upper lip. Oral Surg Oral Med Oral Pathol Oral Radiol Endod 2009;108(5):e51-e53.

[34] Just P-A, Miranda L, Elouaret Y, Meatchi T, Hans S, Badoual C. Classification des tumeurs des glandes salivaires. Ann Otolaryngol Chir Cervicofac 2008;125:331-40.

[35] Steele NP, Wenig BM, Sessions RB. A case of pleomorphic adenoma of the parotid gland metastasizing to a mediastinal lymph node. $\mathrm{Am}$ J Otolaryngol 2007;28(2):130-3.

[36] Altemani A, Martins MT, Freitas L, Soares F, Araújo NS, Araújo VC. Carcinoma ex pleomorphic adenoma (CXPA): immunoprofile of the cells involved in carcinomatous progression. Histopathology 2005;46:635-41.

[37] Cheuk W, Chan JKC. Advances in salivary gland pathlogy. Histopathology 2007; 51(1):1-20.

[38] Ihrler S, et al. Intraductal carcinoma is the precursor of carcinoma ex pleomorphic adenoma and is often associated with dysfunctional p53. Histopathology 2007;51(3):362-71. 